Aharoni, Asaph

\section{Strawberry on chips: gene expression analysis during strawberny development using CDNA microarrays}

\author{
Asaph Aharoni' ${ }^{1}$, Paul L.C. Keizer', Mark Schena', Harrie A. \\ Verhoeven ${ }^{1}$, Arjen J. van Tunen ${ }^{1} \&$ Ann $0^{\prime}$ Connell $^{1}$
}

\author{
${ }^{1}$ Department of Cell Biology, DLO-Centre for Plant Breeding and Reproduction \\ Research, Wageningen, The Netherlands \\ ${ }^{2}$ Centre for Biometry Wageningen, The Netherlands \\ ${ }^{3}$ Department of Biochemistry, Beckman Center, Stanford University M edical \\ Centre, Stanford, California 94305, USA
}

Strawberry fruit have high economic value for both the fresh market and the food industry. During fruit maturation, dramatic changes occur which influence traits such as firmness, pigmentation, sweetness and flavour. We are interested in the isolation of genes, that directly influence strawberry development and ripening. Novel genes identified will be further used to genetically engineer strawberries with improved quality traits. The first step involved the cytological and biochemical analysis of the strawberry fruit. The second step entailed the construction of a ripe strawberry fruit cDNA library. From this library 1,800 cDNAs were randomly selected and spotted in high density on microscope slides. Hybridization experiments comparing gene expression in the green, white, turning and red stages of fruit development identified more than 200 differentially expressed cDNAs. Clustering the genes into functional groups showed that metabolic pathways concerned with secondary metabolism were evident at later stages of development. This is in perfect agreement with physiological observations noted previously. Results demonstrate that cDNA microarrays provide a powerful tool for the comprehensive investigation of the temporal expression pattern of genes relating to strawberry fruit development. The pattern of gene expression within a cell can provide strong clues to their biological role and provide information concerning the physiological state of the cell.

\section{Albertson, Donna}

\section{Measurement of DNA sequence copy number variation using comparative genomic hybridization to microarrays}

\author{
D.G. Albertson ${ }^{1,2}$, R. Segraves ${ }^{2}$, B. Huey², X. Zhang ${ }^{2}$, J. Palmer², S. \\ Blackwood $^{1}$, A. Snijders ${ }^{1}$, G. Hamilton ${ }^{2}$, B. Ljung ${ }^{3}$, S. Dairkee ${ }^{4}$, L. \\ Bolund $^{5}$, H. Yuang 5 , E. Niebuhr ${ }^{6}$ J.W. Gray ${ }^{2} \&$ D. Pinkel ${ }^{2}$ \\ ${ }^{1}$ Cancer Research Institute, ${ }^{2}$ Cancer Genetics Program and ${ }^{3}$ Department of \\ Pathology, University of California, San Francisco, San Francisco, California, USA \\ ${ }^{4}$ Geraldine Brush Cancer Research Institute, San Francisco, California, USA \\ ${ }^{5}$ Institute of Human Genetics, Aarhus University, Aarhus, Denmark \\ ${ }^{6} \mathrm{M}$ edical Genetics, University of Copenhagen, Copenhagen, Denmark
}

Comparative genomic hybridization using arrays of genomic cosmid, P1 and BAC clones (array $\mathrm{CGH}$ ) provides quantitative copy number data over a wide dynamic range with resolution determined solely by the size and genomic spacing of the arrayed clones. We have demonstrated previously that array CGH has the measurement precision to reliably discriminate single copy-level changes from diploid in the human genome. We are currently using this capability to map the extent of deletions on chromosome $5 \mathrm{p}$ in Cri du Chat patients and find fluorescence ratios for diploid targets of $1.0 \pm 0.07$, while ratios on deleted clones are $0.55 \pm 0.05$ (mean \pm s.d.). This measurement precision is expected to permit recognition of deletions with false-positive and -negative rates below one in several thousand. Recently, we have developed procedures that adequately suppress hybridization from repeat sequences in mouse genomes so that heterozygous deletions and duplications can now be mapped with the same accuracy in interspecific backcross animals. In the course of these studies, one clone in mouse and one in human were found to have a ratio of approximately 0.75 when included in the deletion. FISH mapping showed that these clones hybridized to two locations in the genome. Thus, the measured ratios on these clones reflect the accurate measurement of the copy number decrease from 4 to 3 copies in the deletions.

The unprecedented high dynamic range and quantitative accuracy of array CGH also provide the capability to very precisely map copy number profiles across an amplified region using contiguous and overlapping clones as the array elements. When such an analysis was performed across a 1-Mb contig at 20q13.2, we observed a constant level of elevated copy number across the region in some tumours, whereas we recorded abrupt variations in copy number in others. The boundaries of different levels of amplification were thus mapped to within a fraction of a BAC or P1 clone. In some tumours, copy number profiles showed narrow peaks of amplification (some as small as $\sim 300 \mathrm{~kb}$ ) and revealed the presence of two separate regions in the $1-\mathrm{Mb}$ contig that could be the most highly amplified in different tumours. This information focuses attention on the genes mapping to the peak regions and may aid in the identification of the candidate driver oncogenes. These studies using array CGH illustrate the capability of this technology for scanning the entire genome for copy number aberrations, identifying disease genes and providing diagnostic information.

Alizadeh, Ash

\section{Analysis of gene expression in normal and malignant lymphocytes using the Lymphochip cDNA microarray}

\author{
Ash Alizadeh ${ }^{1,2}$, Michael B. Eisen'1, R. Eric Davis², John I. Powell', \\ Liming Yang ${ }^{3}$, Robin R. Hart ${ }^{3}$, Hajeer Sabet², Truc H. Tran², \\ Xin $\mathrm{Yu}^{2}$, Chi Ma², Wing C. Chan ${ }^{4}$, Timothy C. Greiner", \\ Dennis D. Weisenburger ${ }^{4}$, James 0 . Amitage ${ }^{4}$, Izidore Lossos ${ }^{1}$, \\ Ron Levy ${ }^{1}$, David Botstein ${ }^{1}$, Louis M. Staudt ${ }^{2} \&$ Patrick O . Brown $^{1}$ \\ ${ }^{1}$ Departments of Genetics, Biochemistry and M edicine, Stanford University \\ School of M edicine, California, USA \\ ${ }^{2} \mathrm{M}$ etabolism Branch, National Cancer Institute, NIH, USA \\ ${ }^{3}$ Biolnformatics and M olecular Analysis Section, CIT, NIH, USA \\ ${ }^{4}$ Departments of Pathology and M edicine, University of Nebraska M edical
}

Center, Nebraska, USA

Genome-wide knowledge of gene expression in cancer cells promises to illuminate many aspects of their clinical behaviour. We have begun a study of gene expression in lymphoid malignancies by constructing a specialized cDNA microarray, termed the 'Lymphochip', that is enriched in genes selectively expressed in lymphocytes and genes regulating lymphocyte function. As most human lymphomas appear to represent malignant transformation of the germinal center B lymphocyte, we created a cDNA library from germinal center B lymphocytes purified by flow sorting from human tonsils. We obtained 50,000 sequences from this library, over $10 \%$ of which had not been observed previously in other libraries. This rich source of novel genes formed the basis of the Lymphochip microarray, which currently contains over 15,000 clones.

Initial experiments with the Lymphochip have focused on two B cell malignancies: diffuse large cell lymphoma, a common and aggressive subtype of nonHodgkin lymphoma, and chronic lymphocytic leukaemia. We chose these malignancies for study because they most likely encompass a variety of molecularly distinct diseases that cannot be distinguished morphologically. Disease-specific sets of genes were identified that were characteristically expressed in all cases of one malignancy and not the other. Nonetheless, substantial variation in gene expression was observed between cases in a given diagnostic group, which could 